\author{
Lars Eichler \\ Werner J. Z'Graggen \\ Marco Caversaccio \\ Matthias Sturzenegger
}

\section{Symptomatic epilepsy with a tumor in the nose}

Received: 4 November 2005

Received in revised form: 16 February 2006 Accepted: 28 February 2006

Published online: 19 June 2006

Sirs: In adults a meningoencephalocele/encephalocele is a very rare cause for an intranasal tumor. Mostly diagnosis can be made by high resolution (HR) CT and MRI $[1,2]$. The former gives detailed images of skullbase osseous defects, while the latter helps in differentiating herniated brain from neoplasms, in particular meningeoma, esthesioneuroblastoma or carcinoma of the paranasal sinuses. Nevertheless, in some cases histological sampling is advisable to reach definite diagnosis. Because of possible complications such as cerebrospinal fluid (CSF) leakage, chronic sinusitis or recurrent meningitis surgical therapy has to be considered.

A 55-year-old, morbidly obese woman (BMI: $48.5 \mathrm{~kg} / \mathrm{m}^{2}$ ) was brought to the emergency department having suffered a series of 7

L. Eichler · W.J. Z'Graggen

Prof. Dr. M. Sturzenegger ( $\varangle)$

Department of Neurology

Inselspital

3010 Berne, Switzerland

Tel.: + 41-31/632 4837

Fax: +41-31/632 3381

E-Mail: matthias.sturzenegger@insel.ch

M. Caversaccio

Department of ORL, Head and Neck

Surgery

Inselspital

University of Berne, Switzerland generalized epileptic seizures. Upon arrival another generalized, tonic-clonic seizure occurred and the patient had to be orotracheally intubated. CT of the head showed an atrophic right frontal lobe and a, sharply confined and partially calcified mass measuring approximately $3.5 \times 1.8 \mathrm{~cm}$ in the right nasal cavity rather displacing than destroying neighbouring structures. MRI showed the mass to be isodense to brain parenchyma in connection with the frontal lobe (Fig. 1A-C). CSF analysis ruled out coinciding infection. After i.v. valproate had been started in the emergency room no further seizures occurred. The following day, EEG showed no seizure activity. Endoscopic inspection showed a non-pulsating firm mass and a transnasal biopsy was taken without any subsequent complications (Fig. 1D). Histology showed a surface of upper airway epithelium with signs of chronic inflammation and areas of squamous cell metaplasia covering various neurons embedded in glial tissue without surrounding meningeal tissue. There were no signs of cellular atypia or neoplastic transformation. Based on these findings diagnosis of encephalocele could be confirmed. Detailed history contained no clues for recurrent episodes of CSF rhinorrhea or meningitis. The patient was involved in a car accident at the age of 33 years, resulting in a fracture of the third cervical vertebra accompanied by nasal hemorrhage. As a likely trigger of this first episode of epilepsy a two-day sleep withdrawal could be identified.

Meningoencephaloceles and encephaloceles occur as acquired lesions following surgical, traumatic, neoplastic or infectious destruction or as congenital malformations of the skull. The latter are considered disraphic anomalies and show predisposed localisations explained by the embryological development of the skull. Congenital meningoencephaloceles protruding into the nasal cavity through the ethmoidal bone represent an exception, having a higher incidence in eastern asia [3, 4]. Meningoencephaloceles at the the skullbase tend to stay undetected. These occult malformations present on CT or MRI as accidental findings or after becoming apparent by CSF rhinorrhea, recurrent meningitis (typically caused by upper-airway bacterial flora), chronic nasal obstruction or recurrent sinusitis. Seizures as first clinical presentation are very rare.

Although a congenital malformation cannot be excluded, a traumatic origin of the diagnosed encephalocele is most likely [5]. This assumption is supported by the fact that there was no proof of surrounding meningeal tissue. Furthermore localisation at the cribriform plate is suggestive. Relevant head trauma may have resulted from the reported accident and CSF leakage may have been occult or missed in the context of nasal hemorrhage. A secondary progression of an initially small defect is likely, since chronically elevated intracranial pressure, as can occur in obese people, has been shown to be a risk or trigger factor [6].

One has to ask, whether there is a need for surgical therapy. The epileptic seizures were likely to have been triggered by sleep withdrawal. Disturbance of right frontal lobe architecture has to be assumed as a predisposing factor, although EEG (after starting treatment) failed to show focal alterations. Alternatively, inflammatory changes could be the trigger for the epileptic seizures as 
Fig. 1 A) Coronal T1-weigthed MRI showing a dysmorphic right frontal lobe with the appearance of being "dragged down" into the nasal cavity. A partially calcified mass in the right nasal cavity, displaces neighboring bony structures. B) Axial T2weigthed MRI showing volume reduction of the right frontal lobe. C) Sagittal gadolinium-enhanced T1 weighted MRI showing slight enhancement of the approximately $3.5 \times 1.8 \mathrm{~cm}$ intranasal mass in direct communication with the frontal lobe pole. D) Endoscopic view of the intranasal mass shortly after biopsy
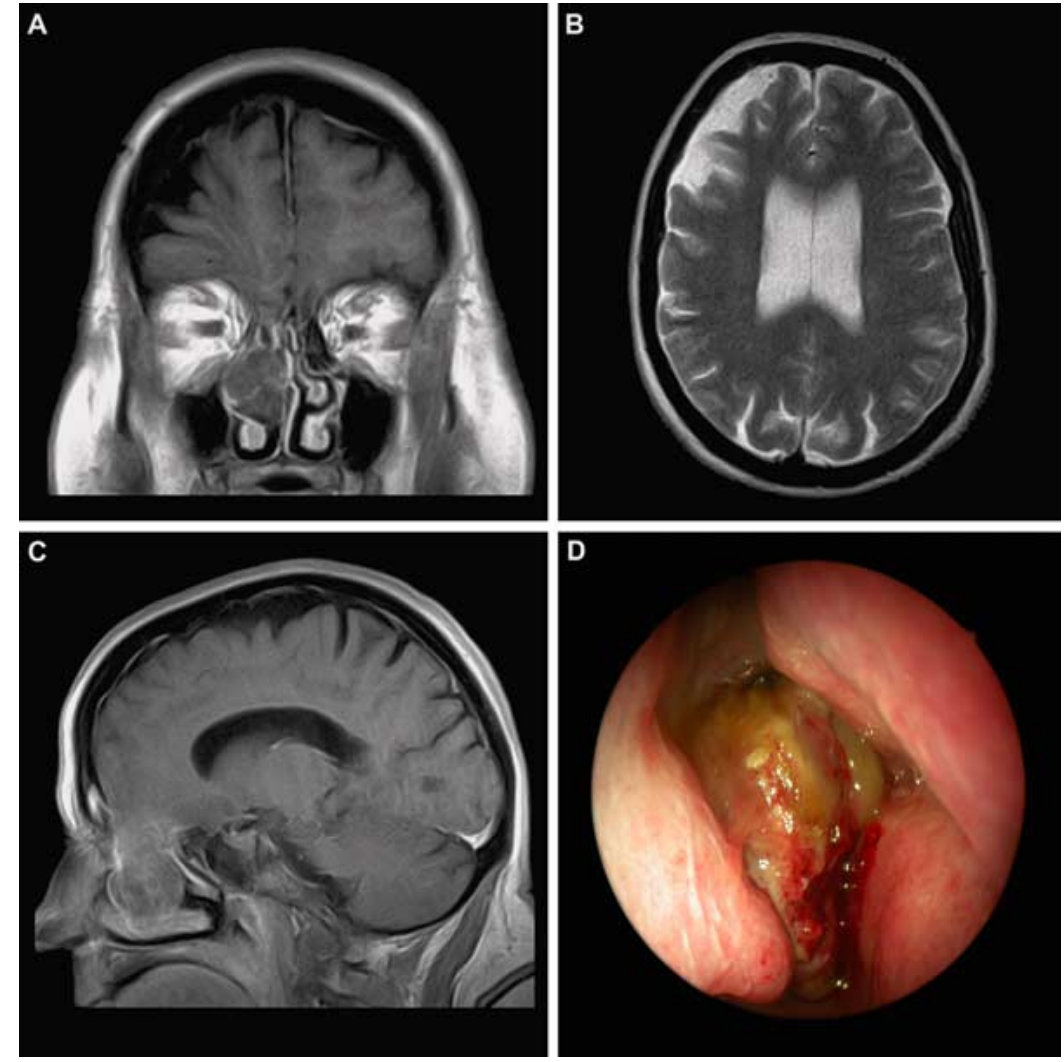

documented by the observed calcifications on CT . Histology confirmed chronic inflammation and highlights the risk of ascending infection (meningoencephalitis). Nevertheless the surrounding upper airway epithelium may provide some protection. Surgical therapy generally is indicated to prevent recurrent infections and would include plastic reconstruction of skullbase defect after excision of the intranasal mass. This procedure probably would not reduce the risk for further epileptic seizures. In this context, the surgical morbidity has to be considered, especially since the patient had a higher surgical risk from obesity. Since no intracranial infection had occurred so far and the patient hesitated to accept surgery, a wait and see strategy including antiepileptic medication and avoidance of possible provoking factors was followed. If CSF rhinorhea or meningitis/ meningoencephalitis occurred surgical therapy would be strongly advised.

\section{References}

1. Horky JK, Chaloupka JC, Putman CM, Roth CM (1997) Occult spontaneous lateral temporal meningoencephalocele: MR findings of a rare developmental anomaly AJNR Am J Neuroradiol 18: 744-746

2. Zinreich SJ, Borders JC, Eisele DW, Mattox DE, Long DM, Kennedy DW (1992) The utility of magnetic resonance imaging in the diagnosis of intranasal meningoencephaloceles Arch Otolaryngol Head Neck Surg 118: 1253-1256

3. Mahatumarat C, Rojvachiranonda N, Taecholarn C (2003) Frontoethmoidal encephalomeningocele: surgical correction by the Chula technique Plast Reconstr Surg 111: 556-567

4. Harris MJ (2001) Why are the genes that cause risk of human neural tube defects so hard to find? Teratology 63: 165-166

5. Talamonti G, Fontana RA, Versari PP, Villa F, D'Aliberti GA, Car P, Collice M (1995) Delayed complications of ethmoid fractures: a "growing fracture" phenomenon Acta Neurochir (Wien) 137: 164-173

6. Meco C, Oberascher G (2004) Comprehensive algorithm for skull base dural lesion and cerebrospinal fluid fistula diagnosis Laryngoscope 114: 991-999 